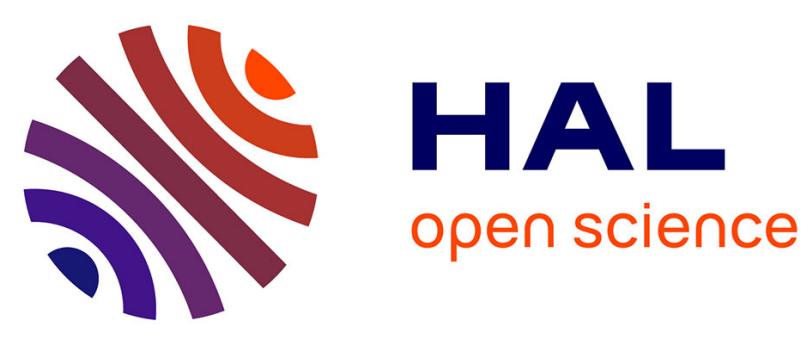

\title{
Kukrit Pramoj (1911-1995)
}

François Lagirarde

\section{To cite this version:}

François Lagirarde. Kukrit Pramoj (1911-1995): Artiste, banquier, écrivain, homme politique et journaliste thaïlandais. Bulletin de l'Ecole française d'Extrême-Orient, 1996, 83 (1), pp.259-264. 10.3406/befeo.1996.2413 . halshs-02569759

\section{HAL Id: halshs-02569759 \\ https://shs.hal.science/halshs-02569759}

Submitted on 11 May 2020

HAL is a multi-disciplinary open access archive for the deposit and dissemination of scientific research documents, whether they are published or not. The documents may come from teaching and research institutions in France or abroad, or from public or private research centers.
L'archive ouverte pluridisciplinaire HAL, est destinée au dépôt et à la diffusion de documents scientifiques de niveau recherche, publiés ou non, émanant des établissements d'enseignement et de recherche français ou étrangers, des laboratoires publics ou privés. 


\section{Kukrit Pramoj (1911-1995)}

Artiste, banquier, écrivain, homme politique et journaliste thaïlandais François Lagirarde

\section{Citer ce document / Cite this document :}

Lagirarde François. Kukrit Pramoj (1911-1995). In: Bulletin de l'Ecole française d'Extrême-Orient. Tome 83, 1996. pp. 259-264; doi : https://doi.org/10.3406/befeo.1996.2413

https://www.persee.fr/doc/befeo_0336-1519_1996_num_83_1_2413

Fichier pdf généré le 08/02/2019 


\section{Kukrit Pramoj 1911-1995}

Artiste, banquier, écrivain, homme politique et journaliste thaïlandais

\section{François LAGIRARDE}

Mom Rajawong Kukrit Pramoj (prononcer Pramôte) s'est éteint le 9 octobre 1995 à l'âge de 85 ans. Ses succès et ses échecs, ses talents et ses libertés, son humour et ses emportements l'avaient rendu immensément populaire dans tout le royaume de Thaïlande. Kukrit fut, parmi les personnalités thaïes de ce siècle, un modèle d'esprit dynamique, tantôt traditionaliste, tantôt non-conformiste, toujours respecté, sinon apprécié. Après une carrière politique saccadée, suivie d'un long intermède consacré aux arts et aux lettres, il devint une véritable institution nationale, une éminence grise des cercles politiques (à dire vrai, une éminence haute en couleur) dont l'influence demeura considérable jusqu'à la fin de sa vie. C'est ainsi que, ces vingt dernières années, de sa retraite bien connue du soï Suan Plu, Kukrit continuait à se prononcer avec force et humeur sur les sujets passionnant l'opinion publique. Même lorsqu'il n'occupait plus aucune fonction, officielle ou privée, ténors du monde politique, magnats de la finance et de l'industrie, simples citoyens et petits paysans venaient consulter ce «sage», aussi bien sur des affaires d'État que pour des problèmes personnels. Kukrit était une voix qu'on écoutait. Une voix qui, dans le système des relations sociales tel qu'il est vécu par les Thaïs, continuait d'exercer un réel pouvoir.

La disparition de Kukrit Pramoj ne manquera pas d'être tristement ressentie, particulièrement dans la classe politique thaïlandaise dont les membres, contrairement à celui-ci, ne savent pas toujours très bien sourire de leurs contradictions ni même parfois en prendre pleinement conscience.

Kukrit est né sur le bateau qui emmenait sa mère à Phitsanulok par la Ménam Chao Praya, le 20 avril 1911. Il était le fils du prince Khamrob et de Mom Daeng et l'arrièrepetit-fils du roi Rama II. Kukrit fut toujours fidèle à ses nobles origines ainsi qu'à la singularité symbolique de sa naissance : sous les lumières de ces bonnes étoiles ont grandi un Siamois par excellence et un original très tôt remarqué.

En effet, élevé dans l'entourage royal, Kukrit fait vite l'usage de ses dons précoces et de son esprit aussi vif que malicieux : il étonna doublement, dit-on, le roi Vajiravudh (Rama VI) en répondant sous un pseudonyme à une difficile question de scolastique bouddhique lors d'un concours présidé par le souverain. Après avoir reçu une excellente éducation dans les écoles du Siam, il partira à l'âge de quatorze ans pour l'Angleterre où il obtiendra un B.A. à Oxford, en philosophie, politique et économie.

Il revient à Bangkok en 1933, un an après l'établissement de la monarchie constitutionnelle. Il devient fonctionnaire, puis, en 1936, occupe un poste important à la Banque commerciale du Siam. Il quittera ce dernier établissement pour fonder, avec un groupe d'associés, la Banque de Thaïlande. Marié à cette époque, Kukrit ne sera guère 
un époux exemplaire et divorcera sept ans après son mariage pour demeurer célibataire jusqu'à la fin de ses jours.

En 1941, il est «enrôlé» (c'est le terme qu'il utilisera lui-même plus tard) pour combattre les troupes «franco-lao » sur les zones frontalières disputées à l'Union française qui seront prises et rétrocédées en 1946 : il se bat sur le Mékong qu'il franchira lors de l'occupation de la ville laotienne de Ban Houei Xai. Il gardera un souvenir vaguement douloureux et plutôt amusé de ces «incidents » que l'histoire n'a pas mis à l'actif de la Thaïlande. Il est vraisemblable que Kukrit ait eu à cette époque une certaine sympathie pour les doctrines «pan-thaïes» favorisées par les «alliés» japonais et qu'il se soit volontairement engagé - il avait alors trente ans - dans les armées de Phibul Songkhram. Pour la première fois dans sa vie, il s'oppose clairement à son frère aîné, Seni, qui, au même moment, à Washington, appelait les Thaïlandais à la résistance...

Revenu du front, Kukrit enseigne à l'Université Thammassat puis à Chulalongkorn. En 1945 il se lance dans la politique sur les traces de Seni, devenu Premier ministre la même année. Kukrit fonde son propre parti (Parti du Progrès) et, à la suite des élections législatives, emporte l'une des cinq députations de Bangkok. Le Parti du Progrès sera intégré, l'année suivante, au tout nouveau Parti démocrate de Seni dont Kukrit occupera le poste de secrétaire général.

En 1947, Kukrit participe au gouvernement de Khuang Aphaiwong dont il est l'un des ministres sans portefeuille. Après les élections de 1948, il est repris par le même Premier ministre comme ministre des Finances, et, lorsque Phibul Songkhram luimême fera son retour l'année d'après, il devient, mais pour un mois seulement, viceministre du Commerce. Pendant une période de presque dix ans Kukrit prendra alors ses distances avec l'action politique directe, le temps que Phibul soit finalement obligé de partir en exil à la suite d'un nouveau coup d'État. Kukrit en profite alors pour créer le quotidien Siam Rath qui, rapidement, parviendra à un très gros tirage. Il utilisera doublement cet organe de presse : d'abord pour exprimer ses critiques et ses commentaires sur le monde politique et sur la société thaïe dans des éditoriaux souvent virulents, ensuite pour publier son œuvre littéraire qu'il livrera sous la forme de feuilletons quotidiens. De cette époque datent ses principaux romans.

Kukrit se consacre ensuite, nouvelle phase de sa vie, aux œuvres sociales et devient le président d'une importante fondation d'assistance aux enfants défavorisés. Il accepte de tourner en 1963 dans une production hollywoodienne (The Ugly American, avec Marlon Brando) jouant le rôle du Premier ministre d'un État asiatique imaginaire menacé par l'insurrection communiste; fiction que la réalité allait "curieusement » rejoindre une douzaine d'années plus tard. Se dirigeant de plus en plus vers l'expression artistique, Kukrit reprend l'étude du théâtre masqué et de la danse classique siamois (khon). Il fonde la troupe de khon de Thammassat, puis, dans la même université, l'Institut des études thaïes sans manquer de donner une partie de son temps pour enseigner à la faculté des Arts libéraux.

En 1968, Kukrit, nommé sénateur, effectue un retour discret dans le monde politique; il occupe toujours ce poste lorsqu'en octobre 1973, les «Trois Tyrans» (Thanom et Narong Kittikachorn, Praphas Charusathien ${ }^{1}$ ) tentent d'écraser par la répression la plus brutale le mouvement étudiant. Forcés à l'exil, ces trois hommes forts laissent un pays en état de choc mais dont une bonne partie de la population semble bien décidée à trouver la voie de la démocratie. Kukrit fera partie des dix-huit membres du comité chargé de rédiger une nouvelle constitution capable de répondre aux aspirations du moment. Celle-ci aurait été sans doute la plus libérale des chartes thaïlandaises si elle avait eu la chance d'être acceptée dans sa forme originale. Hélas, ce

1. Figure parfaite du népotisme : Thanom était le père de Narong et Narong le gendre de Praphas. 
ne fut pas le cas. Kukrit devient le président de l'Assemblée législative chargée d'accepter ou de rejeter ce document : mais cette assemblée, élue par une convention nationale dont les membres furent manipulés et remanipulés par les vétérans de la classe politique ne sera qu'une sinistre copie de la précédente. La constitution est reprise, transformée et enfin approuvée en octobre 1974. Dans l'intervalle, Kukrit a quitté le Parti démocrate et fondé son propre parti (Parti d'Action sociale) à la tête duquel il obtiendra 18 sièges aux élections législatives de 1975. Celles-ci voient la victoire des démocrates et donc de son frère qui devient Premier ministre.

Mais la coalition de «centre-gauche » dirigée par Seni Pramoj est un échec. À la tête de son parti (Action sociale), Kukrit parvient à constituer un gouvernement de «centre-droit » regroupant rien moins que dix-sept tendances politiques et devient alors le dix-huitième Premier ministre du royaume. Kukrit, farouchement anticommuniste (ses attaques contre le leader gauchiste Tirayudh Boonmee menées dans les colonnes du Siam Rath furent particulièrement féroces), aidé par un appareil politique fait de technocrates et de capitalistes conservateurs, réalisera curieusement une performance politique assez libérale.

C'est ainsi que, malgré sa fragilité, cette coalition fut d'abord couronnée de succès grâce, en particulier, à l'application de certaines mesures populaires : réformes sociales (salaire minimal garanti, aide au développement rural), limitation des mesures anticommunistes qui avaient conduit l'armée à commettre de nombreuses atrocités, accord sur le retrait des troupes américaines de Thaïlande, reprise des relations diplomatiques avec la Chine de Mao. En fait, Kukrit, faisant de trop habiles compromis, se place dès lors au milieu d'une poudrière qui allait exploser en octobre 1976. D'un côté les organisations de droite se renforcent et l'armée (tenue en partie par le général Kriangsak Chomanan, le futur putschiste de 1977) commence à agir en dépit de ses instructions tandis que, de l'autre côté, les étudiants progressistes, de plus en plus actifs, expriment ouvertement leur méfiance pour le chef du gouvernement et se font l'écho d'une certaine grogne venue des petits paysans spoliés.

En janvier 1976, alors que sa coalition connaît les pires remous venus de l'intérieur, Kukrit dissout le parlement et appelle à de nouvelles élections pour le début d'avril. Cette campagne électorale, aux enjeux capitaux, fut la plus violente de toute l'histoire de la «démocratie » thaïe : de nombreux meurtres furent commis par l'extrême-droite pour tenter d'intimider les politiciens libéraux et leurs électeurs. Finalement, et pour cause d'abstentionnisme, les urnes rendront un verdict peu convaincant : d'une courte tête, le Parti démocrate se retrouve en première position. Une nouvelle coalition, qui se passe du parti de Kukrit, mais avec tous les autres présents dans la précédente, se reforme. Kukrit perd même son siège de député (remplacé par Samak Sundaravej, un orateur populiste, alors membre du Parti démocrate).

En avril 1976, Kukrit, très amer, laisse la place à son frère Seni. C'est d'abord pour lui le temps du repos (il est véritablement épuisé par les mésaventures qu'il vient de subir) alors que la Thaillande va vivre la crise ${ }^{2}$ d'octobre 1976 suivie d'une cascade de huit coups d'États (plus ou moins «réussis») qui ne s'arrêtera qu'en 1991. En 1976 Kukrit prend une position de repli dans sa célèbre maison du soï Suan Plu et fait «sa » politique à distance. Il sera cependant réélu député en 1979 et en 1983 dans la même circonscription qu'autrefois. Son propre parti, ayant réalisé le meilleur score à ces dernières élections, obtiendra quinze portefeuilles sur les quarante-quatre disponibles dans le nouveau cabinet de Prem Tinsulanonda. Mais Kukrit, qui aurait pu négocier un ministère important, restera volontairement dans l'ombre. Il quitte la direction du Parti

2. Le 6 octobre, les étudiants « contestataires » sont encerclés et massacrés dans l'université Thammassat par des groupes d'extrême-droite protégés par la police et l'armée. Seni donne alors piteusement sa démission et laisse la place aux militaires. 
d'Action sociale en 1985 ; celle-ci reviendra alors à Sit Sawetsila, puis, jusqu'à ce jour (1996) à Montri Phongphanit.

Cette biographie sommaire de Kukrit Pramoj nous permet de comprendre combien son action politique, au sens large, fut importante et variée. Il n'est cependant pas très facile d'en apprécier l'exacte valeur dès lors qu'on utilise les normes et modèles occidentaux : juger d'un parcours et de son cadre de référence idéologique (définir une couleur ou une théorie) demeure, dans le contexte historique thaï, une véritable gageure. Le problème n'est d'ailleurs pas particulier à Kukrit mais se pose en général pour tous les acteurs de la scène politique thaïlandaise : au-delà des proclamations fidèlement monarchistes et constitutionnelles, au-delà du terme de «démocratie » (toujours utilisé dans le sens de «représentation parlementaire») et des proclamations de foi dans le développement, il est rare d'entendre des opinions ou d'observer des actions qui n'obéissent pas au pragmatisme et à l'opportunisme les plus élémentaires. La culture thaïe n'a reçu ni l'héritage d'une pensée sociale rigoureuse, ni l'influence de mouvements syndicaux ou politiques de grande ampleur, attachés à des doctrines de caractère international. Dans ce flottement des références, comment évaluer les performances nationales et établir des responsabilités ? ${ }^{3}$

Ainsi la politique est-elle considérée, en Thaïlande plus qu'ailleurs, comme un jeu pas très propre qui ne convient pas à tous (en thaï "faire " de la politique se dit précisément «jouer», len). D'où la distance affichée de certains groupes sociaux, l'aristocratie, le clergé bouddhique, et, dans une certaine mesure, souvent paradoxale, l'armée. Les engagements et les «sorties» de Kukrit, arrière-petit-fils de roi, homme de culture internationale, bouddhiste convaincu et citoyen à l'abri de tout soupçon (de corruption) témoignent de ses désirs et de ses réticences profondes vis-à-vis de ce problème. Son parcours fut donc exceptionnellement irrégulier : tenté d'agir, et parfois en dépit de toute cohérence, il se mit au cœur d'implacables contradictions; sa façon de traiter des aspirations démocratiques et populaires en 1976 le montre clairement. Tenté par la fuite, il a tourné à plusieurs reprises le dos à la scène politique pour s'investir dans d'autres activités.

Par ailleurs, l'absence, en Thaïlande, d'une classe de grands commis de l'État, voire l'absence d'État tout court (ou la multiplication d'États dans l'État) est un mal que Kukrit a profondément ressenti et nul doute qu'il ait tenté de donner l'exemple d'une attitude possible face à ces problèmes, une attitude presque désespérée. Entre le pouvoir des fonctionnaires, administrateurs civils, technocrates d'un côté et la force des militaires de l'autre, il est vrai que le développement des partis politiques s'est fait sur un «espace » extrêmement réduit, totalement dépendant de l'aide financière du secteur privé.

En dépit de son authentique stature d'homme public et de son évidente bonne volonté, Kukrit n'a pas échappé au plaisir de mettre son talent au service des manœuvres les moins justifiables : son action politique en 1975 et 1976 se fonde sur l'art d'accommoder les contraires (satisfaire à la fois l'armée et les étudiants contestataires) et de manipuler les forces divergentes. Ce génie éphémère lui apporta les premiers succès de son invraisemblable coalition gouvernementale, bientôt suivis d'une catastrophe sociale sans précédent.

3. Les programmes des partis politiques thaïs sont le meilleur exemple de cet impressionnisme ambiant : aux élections législatives de 1995 aucune plate-forme ne se distinguait fondamentalement d'une autre et le choix s'est donc fait sur des individus (soit parce que le candidat offrait, en termes de corruption, plus qu'un autre, soit, au contraire, parce qu'il présentait une honnêteté suffisante en soi). Ces élections ont vu la victoire du champion des relations patron-client et du népotisme bon enfant, Banharn Silapa-archa. 
Pour finalement donner un verdict d'Occidental, il faut avouer que, malgré un sens critique aigu et des talents d'orateur et de pamphlétaire remarquables, malgré la «simplicité » de son style de vie et malgré son charme personnel, Kukrit demeure politiquement un conservateur populiste, un nationaliste et un technocrate lié à la haute finance et au monde des affaires. Il n'a jamais renié son attirance pour l'idéologie panthaïe ni sa foi dans les vertus du capitalisme plutôt sauvagement appliqué dans la société thaïlandaise. C'est pendant les années où son action était la plus sensible que la Thaïlande se préparait à devenir un des «dragons » de l'Asie du Sud-Est, au prix d'une exploitation éhontée des ressources humaines et d'une spéculation foncière qui est en train de modifier profondément la structure sociale du pays. L'aventure politicienne de Kukrit s'est trop souvent passé d'éthique sociale et son parti (Action sociale) est devenu une mécanique de pouvoir, présente, jusqu'à ce jour, dans toutes les coalitions gouvernementales. Personne $n$ 'attend plus de cette formation politique qu'elle vienne combattre à la racine les trois fléaux qui minent la société thaïe : le manque de mobilité sociale, le colonialisme intérieur et le matérialisme excessif.

Enfin, il ne faut pas oublier de signaler que Kukrit, encore une fois en dépit de son «style» et de ses opinions (sur l'argent ou sur la menace culturelle américaine par exemple) fut, à un moment ou un autre, directeur ou membre du conseil d'administration de la compagnie Pepsi Cola, de l'hôtel Indra, de la Siam Commercial Bank, et de la société de publication Siam Rath.

Reste Kukrit l'écrivain. Son œuvre romanesque, dont une partie a été traduite en anglais, est imprégnée de sa connaissance profonde et harmonieuse de l'histoire thaïe, du bouddhisme, de la pensée et de la littérature occidentale. En Thaïlande cette combinaison demeure finalement assez rare et, lorsqu'on la rencontre, elle est souvent tellement déséquilibrée qu'elle en devient caricaturale d'un côté ou de l'autre...

Avec un style spirituel et une précision remarquable dans l'expression Kukrit a charmé des lecteurs avides de pages de qualité, tout particulièrement avec son œuvre maîtresse, Si Phaen Din (Les quatre règnes), qui racontent la vie de Mae Phloi et de sa famille dans le cadre historique du Siam de Rama V et VI (monarchie absolue) puis du Siam de Rama VII et VIII (monarchie constitutionnelle, dictature militaire, seconde guerre mondiale). Les héros de cette saga familiale incarnent les forces qui s'affrontent à ces divers moments et témoignent psychologiquement des tendances contradictoires au sein des classes dirigeantes de la société siamoise (Phloi est née à la cour du roi et appartient à une famille de bonne noblesse). Si le propos est intéressant en soi il apparaît cependant extrêmement décalé, dans sa problématique et son style, par rapport aux productions littéraires internationales de l'après-guerre. Si Phaen Din n'eut donc aucun succès en dehors de la Thaïlande et des siamisants.

En revanche, ce manque d'universalisme et de vision moderne ne manque pas au deuxième «best seller» de Kukrit : Pai Daeng (Bambou rouge) qui fait dialoguer un chef de monastère traditionaliste (qui bavarde en direct avec le Bouddha) et un chef de village communiste... désaccord puis alliance finale contre d'authentiques gredins ! Une nouvelle fois, mais ici pour cause de plagiat (on aura reconnu le célèbre Don Camillo d'Antonio Guareschi) le succès du livre demeura strictement local. Kukrit n'hésitera pas à persévérer dans la voie de la «contrefaçon » (celle-ci demeure encore, dans des spécialités plus industrielles, l'un des problèmes que la Thaïlande pose à ses partenaires étrangers) et fera prestement passer en siamois certaines œuvres d'écrivains anglosaxons tels qu' Oscar Wilde, John Wyndham et Thornton Wilder.

En littérature comme en politique, un malin génie a donc poussé Kukrit à la facilité par simple désir de plaire, vite et sûrement : cette touchante ingénuité dans la séduction aura été, somme toute, assez efficace. Elle appartient à une Thaïlande du sourire qui ressemble aujourd'hui, sinon à un lieu imaginaire, au moins à une terre du passé. 


\section{BIBLIOGRAPHIE ${ }^{4}$}

\section{Kukrit PRAMOJ}

1961 Red Bamboo, Progress Bookstore, Bangkok.

1981 Si Phaendin (Four Reigns), trad. en anglais par Tulachandra, Duang Kamol, Bangkok.

Kukrit PRAMOJ et Seni PRAMOJ (éd. trad.)

1987 A King of Siam Speaks, The Siam Society, Bangkok.

NEHER, Clark D.

1987 Politics and Culture in Thailand, University of Michigan, Ann Arbor.

Niyom RATHAMARIT

1991 «Le difficile développement des partis politiques en Thaïlande»Intermondes, Revue de l'Université Ramkhamheng, Bangkok, vol. 2, $\mathrm{N}^{\circ} 1$ (3) p.164-169.

Rong SYAMANANDA

1981 A History of Thailand, Chulalongkorn University, Thai Watana Panich, Bangkok.

Vilas MANIVAT et VAN BEEK, Steve

1983 Kukrit Pramoj : His Wit and Wisdom, Duang Kamol, Bangkok.

WYATT, David K.

1981 Thailand: A Short History, Yale University Press, Thai Watana Panich, Bangkok.

WRIGHT, Joseph J. Jr.

1991 The Balancing Act : A History of Modern Thailand, Pacific Rim Press, Oakland, Cal. USA, distribué par Asia Books, Bangkok.

4. Le classement bibliographique des auteurs thaïs (selon les normes thaïlandaises) se fait sur la première lettre du prénom suivi du nom de famille. 